DOI: $10.14451 / 1.192 .18$

\title{
ИЗМЕРЕНИЕ УСТОЙЧИВОГО РАЗВИТИЯ: ОСНОВНЫЕ ПОКАЗАТЕЛИ
}

\author{
(c) 2020 Диденко Мария Павловна \\ Научный сотрудник Института стран Азии и Африки \\ МГУ им. М.В. Ломоносова, Россия, Москва \\ E-mail: Mdidenko7@mail.ru
}

В статье структурированы и проанализированы существующие методики измерения устойчивого развития, выявлены их достоинства и недостатки, определены наиболее перспективные направления исследований в данном направлении. Представлена теория, стоящая за созданием каждого показателя, математическая процедура его составления и его основные особенности. Приведенный анализ помог выявить и структурировать основные достоинства и недостатки существующих методов измерения устойчивого развития, на основе которых были сделаны выводы о проработанности этой темы на современном этапе, а также о наиболее перспективных направлениях дальнейших исследований.

Ключевые слова: устойчивое развитие; экологическая устойчивость, экологическая эффективность; социальная устойчивость, устойчивая конкурентоспособность.

Устойчивое развитие много лет является неотъемлемой частью государственных стратегий самых разных стран мира. Однако до сих пор ведутся споры о содержании этого понятия, теоретическое наполнение концепции продолжает совершенствоваться и по сей день [9]. Многозначность понятия ведет не только к существованию разных трактовок и разработке разных политических программ, но также и обуславливает наличие разных методов и показателей количественной оценки устойчивого развития стран мира.

С одной стороны - эти индикаторы способны продемонстрировать как далеко конкретное государство находится от цели (в том числе и относительно других стран), с другой - их использование затрудняется как отсутствием статистических данных по отдельным составным частям показателя, так и не совсем стройной методологией расчета. Например, для измерения многих компонентов применяется метод экспертных оценок, что неизбежно ведет к росту субъективности индикатора. Тем не менее, подобные недостатки не повод отказываться от попыток составить показатель, который смог бы характеризовать положение страны в контексте устойчивого развития. Далее мы проанализируем несколько таких индикаторов.

Начиная с саммита ООН 1992 г., прошедшего в Рио-де-Жанейро, общепризнанным стало деление устойчивого развития на три части: экономическую, экологическую и социальную
[2]. Оно сохранило свою популярность и сейчас, например, оно предлагается и в резолюции $\mathrm{OOH}$ 2015 г. [14]. Анализируя показатели устойчивого развития, для начала мы будем придерживаться именно этой схемы.

Начнем с индексов, которые задуманы как комплексные показатели, охватывающие все аспекты устойчивого развития. Один из них $-u н-$ декс совокупного благосостояния (Inclusive Wealth Index) [21], он был разработан центром Международной программы гуманитарных аспектов глобальных изменений ООН. В основе его методологии как раз лежит представление о том, что устойчивое развитие состоит из трех основных частей - экономической, экологической и социальной, поэтому именно эти группы индикаторов и были выбраны для расчетов. В своей первой версии в 2008 г. индекс был представлен только для 24 государств. Методология расчетов постепенно менялась, и уже в 2018 году он был рассчитан для 140 стран мира. Характерной особенностью этого показателя является то, что он призван измерить не текущее положение дел в стране, а ее перспективы, поэтому для расчетов используются показатели запасов:

1) Природных: полезные ископаемые, углеводородное сырье, леса, земли, используемые для сельского хозяйства, рыба;

2) Производственных: оборудование, заводы, жилье, инфраструктура;

3) Человеческих: образование.

Эти индикаторы сначала агрегируются, а за- 
тем осуществляется корректировка на наносимый окружающей среде вред от углекислого газа, средства, полученные от продажи нефти, а также на совокупную производительность факторов производства.

Если говорить о недостатках этого индекса, то главным, на наш взгляд, является отсутствие в расчетах показателей социального капитала, а также индикаторов, оценивающих качество институтов. Однако авторы исследования ссылаются на то, что концепции, стоящие за этими понятиями пока еще неокончательно сформированы, а значит включение их в модель пока лучше отложить.

Другой попыткой комплексного измерения устойчивого развития является индекс социального прогресса (Social Progress Index), разработанный группой ученых во главе с М.Портером в 2015 г. Однако в отличие от предыдущего рассматриваемого показателя, он не включает в себя индикаторы экономического развития, таким образом, он состоит из комбинации социальных и экологических факторов. Такой выбор авторов, например, дает возможность проводить регрессионный анализ, где зависимой переменной будет выступать сам индекс, а факторами можно выбрать любые показатели экономического развития.

В основе создания индекса лежит следующее определение социального прогресса - «способность общества удовлетворять базовые потребности его членов, создавать условия и механизмы, способствующие поддержанию желаемого уровня качества жизни и достижению индивидуумами максимума своих возможностей» [19]. Отсюда логично деление на три группы факторов:

1) Базовые человеческие потребности еда и медицинские услуги, доступ к воде и канализации, наличие жилья, безопасность.

2) Благосостояние - доступ к знаниям, к информации и средствам связи, доступ к услугам сферы здравоохранения, качество экологии.

3) Возможности - права, свобода личности и выбора, толерантность в обществе и инклюзия, доступ к высшему образованию.

В каждую из этих категорий были отобраны по 3-5 показателя, методология получения которых соответствует критериям прозрачности, а у статистических данных по всем странам - один и тот же источник. Процедура создания итогового индекса очень проста: каждому показателю придается равный вес, и то же самое делается с вышеуказанными категориями.

На данный момент доступны расчеты для 127 государств мира в 2017 г., по большинству из них значение индекса получено также и за 2014-2016 гг., то есть временной ряд, доступный для сопоставлений -4 года, что с одной стороны - дает представление о возможных тенденциях в изменении показателей, с другой - недостаточно для того, чтобы сделать решительные выводы.

Третий индикатор, который можно поставить в один ряд с вышеописанными - Индекс развития человеческого потенциала, рассчитанный в рамках Программы Развития ООН [18]. Этот показатель включает в себя три компонента: продолжительность жизни, уровень образования и ВВП на душу населения в ППС, который, по задумке авторов, показывает уровень жизни. С одной стороны - методология очень проста и прозрачна, с другой - подобная простота обусловлена в первую очередь отсутствием дополнительных показателей, которые могли бы заметно скорректировать значение индекса для отдельных стран. Например, довольно популярной является вариация Индекса человеческого развития, скорректированная на гендерное неравенство.

К другим попыткам соединить три составляющие устойчивого развития воедино можно отнести индексы устойчивой конкурентоспособности Всемирного экономического форума и корейского агентства SolAbility.

Индекс ВЭФ имел короткую историю и был посчитан только в 2014 г. В его основу была положена идея о необходимости корректировки индекса международной конкурентоспособности на социальные и экологические факторы. Максимальный размер корректировки был задан 20\% [12]. Главной проблемой, с которой столкнулись авторы, и которая во многом предопределила жизнеспособность этого показателя, стало отсутствие разработанной теоретической базы, которая могла бы лечь в основу определения весов для разных компонентов индекса.

Что касается индекса устойчивой конкурентоспособности SolAbility [20], то он рассчитывается и по сей день. Авторы считают, что залог устойчивой конкурентоспособности находится в наличии природных ресурсов, их эффективном использовании, интеллектуальном капитале, социальной стабильности и грамотном го- 
сударственном управлении. При таком взгляде на конкурентоспособность большее внимание уделяется именно ее устойчивости, то есть на передний план выходит то, что в индексе ВЭФ могло составить максимум 20\%. Однако главный недостаток у индекса все тот же - субъективно заданные веса компонентов. Также необходимо добавить, что методология постоянно менялась, поэтому сравнивать результаты в динамике нельзя.

Далее проанализируем показатели, которые посвящены одному из аспектов устойчивого развития, а именно - экологии.

Начнем с индекса экологической эффективноcmu (Environmental Performance Index) Йельского университета [15]. Первоначально в 2000 г. индикатор представлял из себя неструктурированную совокупность 76 показателей, в 2006 г. их количество было сокращено до 22, что сделало индекс более простым и удобным в использовании, также были сняты опасения о возможном двойном учете данных. Дальнейшие изменения касались не только состава показателей, но также и их группировки, выбора процедуры нормализации данных и удельных весов. Доклад, опубликованный в 2016 г. включает в себя не только индекс, рассчитанный для этого года, но также и для 2007-2016 гг., что позволяет анализировать изменение показателя для каждой страны в динамике. В 2018 г. в свет вышел новый индекс экологической эффективности и в силу изменения методологии, он уже несопоставим с индексами предыдущих лет.

Авторы исследования ставят перед собой две цели: с одной стороны - измерить качество экологии, а с другой - ее жизнеспособность. Или, другими словами, предлагается рассмотреть и экологические факторы, влияющие на здоровье человека, и то, насколько эффективно используются природные ресурсы с точки зрения желаемого сохранения экосистем. Исходя из этой задачи, для последней версии индекса в 2018 г. были подобраны 24 статистических показателя, которые затем были сгруппированы в 10 категорий.

На первоначальном этапе составления индекса все показатели приводятся по шкале от 0 до 100, для их трансформации используется алгоритм мин-макс:

значение индикатора = ((показатель страны-нижняя граница) / (верхняя граница-нижняя граница)) × 100\%
Под верхней границей понимается заданный высокий показатель выборки, для его определения прибегают к докладам международных организаций, использованию экспертных опросов или выбирают 95 или 99 перцентиль. Для нижней границы чаще всего используется именно перцентильный критерий. Если показатель страны выходит за установленные рамки, то ему присваивается значение 0 или 100 соответственHо.

Показатели разбиваются на 10 групп, при этом им присваиваются экспертно заданные веса, затем группы агрегируются по двум аспектам (качество экологии и жизнеспособность экосистемы), после чего первый показатель получает множитель 0,4, а второй - 0,6 (этот выбор объясняется разной вариацией компонентов). Необходимо отметить, что у составленного таким образом индекса есть ряд недостатков:

1. Первичные статистические данные сначала проходят процедуру нормализации, а от ее выбора будет меняться и итоговое значение индекса.

2. Все веса задаются методом экспертной оценки, что, хотя и позволяет сохранить прозрачность методологии, делает ее более субъективной.

3. Модель не охватывает многие важные аспекты состояния экологии, например, уровень заболоченности и опустынивания почв.

Другой показатель, представляющий интерес с точки зрения измерения экологической составляющей устойчивого развития - индекс глобальной адаптации (Global Adaptation Index) [6]. Его создатели хотели оценить степень уязвимости стран к воздействию климатических изменений, а также показать насколько страны готовы к этим изменениям.

Индекс состоит из 74 показателей, разбитых на 45 индикаторов, принимающих посредствам процедуры нормализации мин-макс значения от 0 до 1. 36 индикаторов входят в состав группы «уязвимости», индекс которой - их среднее арифметическое. Оставшиеся 9 индикаторов представляют из себя индикаторы готовности, которые в свою очередь делятся на экономические, социальные и факторы государственного управления, а итоговый индекс по направлению - тоже среднее арифметическое всех индикаторов.

Индекс глобальной адаптации получается путем вычитания показателей уязвимости из 
показателей готовности, добавлением к полученной разнице единицы и умножением суммы на 50.

K главным недостаткам индекса можно отнести постоянные изменения в методологии его расчетов, которые делают невозможным анализ изменения показателя в динамике. Также необходимо отметить, что, как и в вышеописанных индексах, экспертно заданные веса не имеют под собой теоретически обоснованной основы.

Некоторые ученые считают, что рассматривать экологическую проблематику отдельно от социально-экономической вообще не имеет смысла [3], поэтому и индекс экологической эффективности, и индекс глобальной адаптации критикуется не только за методологию расчета, но также под сомнение ставится целесообразность их существования.

Мы уже рассмотрели индикаторы, которые претендуют на то, чтобы охватить все аспекты устойчивости, однако здесь стоит упомянуть показатель, который включает в себя комбинацию экономики и экологии - зеленый ВВП. ВВП корректируется на уменьшение запасов природных ресурсов, расходы на охрану окружающей среды и на ее деградацию. Главная проблема, связанная с расчетом этого показателя, заключается в сложности денежной оценки индикаторов состояния экологии.

Теперь рассмотрим проблему социальной устойчивости. Прежде чем переходить к методикам расчетов, необходимо сказать, что на данный момент параллельно существует несколько совершенно разных версий того, что понимается под «социальной устойчивостью». С одной стороны - социологов обвиняют в любви к расплывчатым формулировкам и отсутствии последовательности [2], с другой - сами социологи зачастую сводят эту проблему к философским дискуссиям о том, какое общество нужно сделать устойчивым [4]. Отсутствие единой теоретической базы предопределило и слабую математическую разработку темы. Наиболее исследованной является проблема политической стабильности, поэтому рассмотрим несколько интегрированных показателей ее измерения.

Часть исследований посвящена выявлению факторов, ведущих к политической нестабильности [8]. Однако далее авторы не предлагают каких-либо интегрированных показателей, с помощью которых можно было бы проделать дальнейшие вычисления и наблюдения. Вместе с тем, есть и варианты композитных индексов, как например - индекс несостоятельности/недееспособности государств, который впоследствии был переименован в индекс хрупкости государств (The Fragile States Index) [10].

Этот индекс по задумке авторов призван измерить способность государства сохранять территориальную целостность, а также избегать экономических и социальных кризисов. Методология расчета индекса оставалась без изменений на протяжении нескольких лет, в ее основе лежит метод системного анализа конфликтов. Таким образом, возможны сравнения в динамике, как для показателей одной страны, так и для сравнения стран, однако авторы исследования считают, что основная цель - отслеживать качественные изменения внутри страны, а не сопоставлять ее с другими.

В рамках расчета индекса проводится контент-анализ прессы, правительственных отчетов, научных статей и т.д. Далее эти данные агрегируются, при этом особое внимание уделяется сравнению с предыдущим годом: проблемам, которые появились в предыдущие периоды, проблемам, которые остались нерешенными. При этом также используются и статистические базы, то есть привлекается достаточно широкий круг источников, что позволяет компенсировать возможное низкое качество отдельных из них.

Далее данные делятся на 100 показателей, которые объединяются в 12 индикаторов, им присваивается количественная оценка от 0 (абсолютная стабильность) до 10. Итоговый индекс получается путем сложения всех этих индикаторов. Страны с наименьшим значением индекса признаются более стабильными. Всего индекс рассчитан для 176 стран мира.

Больше всего вопросов вызывает процедура агрегирования его компонентов - у нее отсутствует прозрачность, что на фоне не самых ожидаемых результатов для отдельных стран, является ощутимым недостатком.

Стоит отметить, что, хотя, как мы и говорили, деление устойчивого развития на три компонента, является общепринятым [7], существуют и другие модели, как например - выделение четвертой составляющей, а именно - культуры [13], и пятой - безопасности [5]. Решение добавить эти аспекты мотивируется ситуацией, сложившейся в последние годы в Европейском Союзе: значительный рост числа мигрантов в странах объединения уже не может не учитываться, ког- 
да речь заходит о социальных, политических и экономических проблемах внутри этих государств. Однако, такая постановка вопроса, к сожалению, не имеет математического решения: так как культура и безопасность - такие понятия, количественное измерение которых крайне затруднительно. Например, модель Г. Хофстеде [9] позволяет измерить культурные ценности, присущие тому или иному государству, однако при этом все расчеты производятся на основе предположения, что культура практически не изменяется во времени, то есть ими невозможно воспользоваться для того, чтобы оценить влияние мигрантов на культуру.

Концепция устойчивого развития продолжает развиваться, а вместе с изменениями теоретической составляющей, меняются и методы его количественной оценки. Самым популярным является деление понятия на три компонента экономический, экологический и социальный. Существуют модели измерения как всех частей в совокупности, так и по отдельности.

Желание исследователей учесть как можно больше аспектов устойчивого развития пока приводит к целому ряду проблем и не всегда удачным решениям:

1) Нехватка качественных статистических данных обычно «компенсируется» включением в модель данных, полученных из опросов, а это в свою очередь усиливает субъективность итогового показателя.

2) Появление/отсутствие новых статистических данных либо решается путем экстраполяции показателей на текущий/предыдущий период, что вводит статистическую погрешность в расчеты, либо изменением состава индекса, что делает результаты за разные годы несопостави- мыми друг с другом.

3) Слабо разработанная теоретическая база ведет к отсутствию основы для взвешивания отдельных частей индекса, что влечет за собой использование оценок экспертов, однако вопрос об удельной важности составляющих настолько спорный, что разброс мнений получается достаточно большой, и решение, основанное на голосе большинства, не выглядит убедительным.

4) В поисках оптимума методология расчетов постоянно меняется, что делает невозможными сопоставления по разным временным периодам.

Таким образом, неудивительно, что все интегрированные показатели устойчивого развития подверглись внушительной критике. На наш взгляд, пока не существует более четкого теоретического представления об устойчивом развитии, более предпочтительной является разработка показателей по отдельным его аспектам. Это касается не только композитных индексов, описанных выше, но и простых статистических показателей, учет которых на данный момент оставляет желать лучшего.

Очевидно, что в теоретическом плане гораздо более разработанным является экологический аспект устойчивого развития, во многом именно поэтому и попытки его количественного измерения выглядят более солидно. Например, индекс экологической эффективности по многим параметрам является удачным мерилом качества и жизнеспособности экологии. Однако для уменьшения субъективной составляющей, возможно, авторам стоит рассмотреть другие существующие методы агрегирования и взвешивания компонентов.

\section{Библиографический список}

1. Agenda 21, Programme of Action for Sustainable Development, UN Doc A/Conf.151/26, 1992.

2. Axelsson, R.; Angelstam, P.; Degerman, E.; Teitelbaum, S.; Andersson, K. Social and Cultural Sustainability: Criteria, Indicators, Verifier Variables for Measurement and Maps for Visualization to Support Planning. Ambio 2013, 42, 215-228.

3. Blewitt, J. Understanding Sustainable Development - Earthscan, London, 2008.

4. Boström, M. A missing pillar? Challenges in theorizing and practicing social sustainability. Sustain Sci. Pract. Pol. 2012, 8, 3-14.

5. Bervar M., Bertoncelj A., The Five Pillars of Sustainability: Economic, Social, Environmental, Cultural and Security Aspects, Management International Conference, Pula, Croatia, 1-4 June 2016

6. Chen, C.; Noble, I.; Hellmann, J.; Coffee, J.; Murillo, M.; Chawla, N., University of Notre Dame Global Adaptation Index Country Index Technical Report, Release date: November, 2015

7. Featherstone, $D$. The contested politics of climate change and the crisis of neo-liberalism. AME Int. J. Crit. Geogr. 2013 
8. Goldstone J., «A Global Model for Forecasting Political Instability», American Journal of Political Science 54 no. 1 , 2010, p. 190-208.

9. Hofstede, G. Dimensionalizing Cultures: The Hofstede Model in Context. Online Readings in Psychology and Culture, 2(1), 2011.

10. Messner, J. J., Fragile States Index Annual Report 2019, The Fund for Peace, 2019.

11. Scerri, A., and J. Paul. «Accounting for sustainability: Combining qualitative and quantitative research in developing 'indicators' of sustainability». International Journal of Social Research Methodology, 2010, 13 (1): 41-53.

12. The Global Competitiveness Report 2014-2015, World Economic Forum, Geneva, 2014.

13. UCLG United Cities and Local Governments. 2010. Culture: Fourth Pillar of Sustainable Development (Online unedited ed.). New York: United Nations

14. UN Transforming our world: the 2030 Agenda for sustainable development. Resolution adopted by the general assembly on 25 September 2015 (A/RES/70/1). United Nations, New York.

15. Wendling, Z. A., Emerson, J. W., Esty, D. C., Levy, M. A., de Sherbinin, A., et al. 2018 Environmental Performance Index. New Haven, CT: Yale Center for Environmental Law \& Policy, 2018.

16. https://epi.yale.edu/ (дата обращения: 04.11.2019)

17. https://fragilestatesindex.org (дата обращения: 04.11.2019)

18. http://hdr.undp.org/en/content/human-development-index-hdi (дата обращения: 04.11.2019)

19. https://socialprogressindex.com/methodology (дата обращения: 04.11.2019)

20. http://solability.com (дата обращения: 04.11.2019)

21. https://unenvironment.org/resources/report/inclusive-wealth-report-2018 (дата обращения: 04.11.2019) 